\title{
Metformin suppresses the expression of Sonic hedgehog in gastric cancer cells
}

\author{
ZHOU SONG, BO WEI, CANRONG LU, XIAOHUI HUANG, PEIYU LI and LIN CHEN \\ Department of General Surgery, General Hospital of Chinese PLA, Beijing 100853, P.R. China
}

Received December 31, 2015; Accepted December 9, 2016

DOI: $10.3892 / \mathrm{mmr} .2017 .6205$

\begin{abstract}
The traditional anti-diabetic drug, metformin, has been found to have anticancer effects. The Sonic hedgehog (Shh) signaling pathway is involved in the on cogenesis of gastric cancer. The aim of the present study was to investigate whether metformin has an effect on the Shh signaling pathway in gastric cancer cells. HGC-27 and MKN-45 human gastric cancer cells were treated with metformin at different concentrations and for different durations. Subsequently the mRNA and protein levels of Shh, Smoothened (SMO), and Glioma-associated oncogene (Gli)-1, Gli-2 and Gli-3 were examined using western blot and reverse transcription-quantitative polymerase chain reaction analyses. RNA interference was used to detect whether the effects of metformin treatment on the Shh signaling pathway were dependent on AMP-activated protein kinase (AMPK). The results revealed that the protein and mRNA levels of Shh and Gli-1 were decreased by metformin treatment in the two cell lines in a dose- and time-dependent manner. Metformin also significantly inhibited the gene and protein expression levels of SMO, Gli-2 and Gli-3. The small interfering RNA-induced depletion of AMPK reversed the suppressive effect of metformin on recombinant human Shh-induced expression of Gli-1 in HGC-27 gastric cancer cells. Therefore, metformin inhibited the Shh signaling pathway in the gastric cancer cell lines and the inhibitory effect of metformin on the Shh pathway was AMPK-dependent.
\end{abstract}

\section{Introduction}

Gastric cancer is one of the most common types of malignant cancer worldwide (1). In addition tosurgery, chemotherapy and radio chemotherapy, there is a demand for novel treatment approaches for gastric cancer due to its poor prognosis (2).

Correspondence to: Professor Lin Chen, Department of General Surgery, General Hospital of Chinese PLA, 28 Fuxing Road, Beijing 100853, P.R. China

E-mail: chenlinbj@vip.sina.com

Key words: metformin, Sonic hedgehog signaling pathway, gastric cancer, AMP-activated protein kinase
Metformin is a widely used anti-diabetic drug, which has been reported to exhibit potential anticancer activity (3). Previous studies have shown that metformin administration significantly reduces the incidence and mortality rates of various types of cancer, including gastric cancer (4-6). According to the findings reported by a previous study, patients who were treated with metformin hada lower incidence of gastric cancer, compared with those who did not receive metformin treatment (7). However, the mechanism underlying the anticancer effects of metformin is complex and remains to be fully elucidated. Activation of the AMP-activated protein kinase (AMPK) pathway has been recognized as a key aspect of the anticancer effects of metformin (8).

The Sonic hedgehog (Shh) signaling pathway is one of the most common signal transduction pathways in human cells (9). It is critical in normal cell differentiation and embryonic development (10). However, abnormal activation of the Shh pathway has been identified in several types of human cancer (11). Substantial evidence has shown that the Shh pathway is crucial to the development and homeostasis of gastric glands. In addition, abnormal activation of the Shh pathway has been found toresult in gastric cancer (12-14).

The aim of the present study was to investigate the effects of metformin on the regulation of the Shh signaling pathway in gastric cancer cells. The expression levels of Shh, Glioma associated oncogene (Gli)-1, Gli-2 and Smoothened (SMO) were examined using western blot and reverse transcription-quantitative polymerase chain reaction (RT-qPCR) analyses. The RNA interference technique was also used to detect whether the effects of metformin on the Shh signaling pathway are dependent on AMPK.

\section{Materials and methods}

Cell culture. The HGC-27 and MKN-45 human gastric cancer cell lines were obtained from the Peking Union Medical College (Beijing, China). The cell lines were cultured in 1640 medium containing $10 \%$ fetal bovine serum and penicillin streptomycin (100 mg/l; Invitrogen; Thermo Fisher Scientific, Inc.). The cells were maintained at $37^{\circ} \mathrm{C}$ in a humidified atmosphere with $5 \% \mathrm{CO}_{2}$.

RNA interference. Small interfering RNAs (siRNAs) specific for the AMPK $\alpha 1$ and control siRNA were purchased from Santa Cruz Biotechnology, Inc. (Santa Cruz, CA, USA). The 
cells were seeded into 12 -well plates at a concentration of $3 \times 10^{5}$ cells/well. Following incubation for $24 \mathrm{~h}$, the cells were transfected with the AMPK $\alpha 1$ siRNAs or control siRNA for $48 \mathrm{~h}$ using Lipofectamine ${ }^{\circledR} 2000$ transfection reagent at $37^{\circ} \mathrm{C}$ (Invitrogen; Thermo Fisher Scientific, Inc.) according to the manufacturer's protocol. Following transfection, the cells were treated in serum-free medium for $24 \mathrm{~h}$, and then used in the following experiments. Cells were divided into five groups: HGC-27 cells treated with PBS alone for $12 \mathrm{~h}$ as a blank control at $37^{\circ} \mathrm{C}$; untransfected HGC-27 cells treated with rhShh $(0.5 \mu \mathrm{g} / \mathrm{ml})$ for 12 hat $37^{\circ} \mathrm{C}$; untransfected HGC-27 cells treated with combination of rhShh $(0.5 \mu \mathrm{g} / \mathrm{ml})$ and metformin (5 mM) for $12 \mathrm{~h}$ at $37^{\circ} \mathrm{C}$; HCG-27 cells transfected with siControl and treated with the combination of rhShh $(0.5 \mu \mathrm{g} / \mathrm{ml})$ and metformin $(5 \mathrm{mM})$ for $12 \mathrm{~h}$ at $37^{\circ} \mathrm{C}$; HCG-27 cells transfected with siAMPK and treated with the combination of rhShh $(0.5 \mu \mathrm{g} / \mathrm{ml})$ and metformin $(5 \mathrm{mM})$ for $12 \mathrm{~h}$ at $37^{\circ} \mathrm{C}$.

Western blot analysis. RIPA buffer supplemented with protease inhibitor was used to lyse the cells for western blot analysis. The protein concentrations were determined using an enhanced BCA protein assay kit (Bio-Rad Laboratories, Inc., Hercules, CA, USA). The protein lysates (30 $\mu \mathrm{g} / \mathrm{sample})$ were separated using SDS-PAGE onan 8 or $15 \%$ gel, and transferred onto a nitrocellulose membrane (GE Healthcare Life Sciences, Piscataway, NJ, USA). The membrane was blocked with $5 \%$ non-fat dry milk in Tris-buffered saline (TBS) containing $0.1 \%$ Tween-20 (TBST). The membranes were then incubated with primary antibody at $4^{\circ} \mathrm{C}$ overnight, and subsequently incubated with horseradish peroxidase-conjugated secondary antibodies (dilution, 1:3,000; cat. no. BM2006; Boster Systems, Inc., Pleasanton, CA, USA) for $1 \mathrm{~h}$ at room temperature. The primary antibodies are listed as follows: Rabbit anti-human monoclonal Shh (dilution, 1:1,000; cat. no. EP1190Y; Abcam, Cambridge, MA, USA), rabbit anti-human monoclonal Gli-1 (dilution, 1:1,000; cat. no. AB49314; Abcam), rabbit anti-human monoclonal Gli-2 (dilution, 1:1,000; cat. no. AB26056; Abcam), rabbit anti-human monoclonal Gli-3 (dilution, 1:1,000; cat. no. AB6050; Abcam), rabbit anti-human monoclonal AMPK (dilution, 1:1,000; cat. no. AB32047; Abcam), and rabbit anti-human monoclonal GAPDH (dilution, 1:1,000; cat. no. AB9485; Abcam). The bands were visualized using chemiluminescence (Santa Cruz Biotechnology, Inc.) and exposed on BioMax film (Kodak, Rochester, NY, USA). Image J software (version 1.41; National Institutes of Health, Bethesda, MD, USA) was used for quantitative densitometric analysis.

RT-qPCR analysis. The total RNA was extracted from the cells using anRNeasy mini kit (Qiagen, Inc., Valencia, CA, USA) according to the manufacturer's protocol. cDNA was synthesized using $2 \mu \mathrm{g}$ of RNA with an RT reagent kit (Takara Biotechnology Co., Ltd., Dalian, China) and the cDNA was then used as a template for RT-qPCR analysis. RT-qPCR was performed using $2 \mu \mathrm{l}$ cDNA in a $20 \mu \mathrm{l}$ reaction system with Maxima SYBR-Green qPCR Master Mix (Thermo Fisher Scientific, Inc.) using the Bio-Rad CFX96 Real-Time PCR Detection system (Bio-Rad Laboratories, Inc.). The specific primers (Table I) were designed using Primer Premier software (version, 5.0; Premier Bio soft International, Palo Alto,
CA, USA). The thermal cycling parameters for RT-qPCR reactions consisted of an initial denaturation step at $95^{\circ} \mathrm{C}$ for $10 \mathrm{~min}$ followed by 40 cycles of denaturation at $95^{\circ} \mathrm{C}$ for $15 \mathrm{sec}$, annealing at $55^{\circ} \mathrm{C}$ for $15 \mathrm{sec}$ and extension at $55^{\circ} \mathrm{C}$ for 15 sec. GAPDH was used as the internal control. The qPCR amplification was performed using an ABI Prism 7500 system (Applied Biosystems; Thermo Fisher Scientific, Inc.) and SYBR-Green PCR master mix (Applied Biosystems; Thermo Fisher Scientific, Inc.). Relative mRNA expression was calculated using the delta-delta $\mathrm{Cq}$ method (15).

Statistical analysis. All experiments were repeated at least three times, and representative results are presented. All quantitative data are presented as the mean + standard deviation. Differences between two groups were compared using the two tailed Student's t-test with SPSS 13.0 statistical software (SPSS, Inc., Chicago, IL, USA). $\mathrm{P}<0.05$ was considered to indicate a statistically significant difference.

\section{Results}

Metformin decreases the protein expression levels of Shh and Gli-1 in gastric cancer cells. The effects of metformin on the protein expression of Shh and Gli-1 in gastric cancer cells were examined using western blot analysis. The gastric cancer cells were treated with different concentrations of metformin $(0,1,5$ and $10 \mathrm{mmol} / \mathrm{l})$ for $12 \mathrm{~h}$ or with $5 \mathrm{mM}$ metformin for different durations $(0,3,6$ and 12 h). As shown in Fig. 1A-D, metformin suppressed the protein expression levels of Shh and Gli-1 in a dose- and time-dependent manner in the HGC-27 cells. As shown in Fig. 1E-H, treatment with metformin also suppressed the protein expression levels of Shh and Gli-1 in the MKN-45 cells.

Metformin decreases the mRNA expression levels of Shh and Gli-1 in gastric cancer cells. The effects of metformin on the mRNA levels of Shh and Gli-1 in the gastric cancer cells were determined using RT-qPCR analysis. The gastric cancer cells were treated with different concentrations of metformin $(0,1,5$ and $10 \mathrm{mmol} / \mathrm{l})$ for $12 \mathrm{~h}$ or with $5 \mathrm{mM}$ metformin for different durations (0,3, 6 and $12 \mathrm{~h})$. As shown in Fig. 2A and B, treatment with metformin decreased the mRNA expression levels of Shh and Gli-1 in a dose- and time-dependent manner in the HGC-27 cells. Treatment of metformin also decreased the mRNA expression levels of Shh and Gli-1 in a dose- and time-dependent manner in the MKN-45 cells (Fig. 2C and D).

AMPK is involved in metformin-induced suppression of the Shh signaling pathway in gastric cancer cells. As shown in Fig. 3A, siRNA specific to AMPK $\alpha 1$ effectively depleted the expression of AMPK at the protein level in the transfected HGC-27 gastric cells, compared with the cells transfected with control siRNA. The protein levels of Gli-1 were also examined using western blot analysis. As presented in Fig. 3B, the blank control group was treated with PBS only. Untransfected cells were treated with recombinant human (rh) Shhor the combination of rhShh and metformin. HCG-27 gastric cells transfected with siControlor siAMPK were also treated with a combination of rhShh and metformin. As shown in Fig. 3B and C, treatment with rhShh significantly upregulated the expression of Gli-1, 
Table I. Primers used for reverse transcription-quantitative polymerase chain reaction analysis.

\begin{tabular}{lll}
\hline Primer & \multicolumn{1}{c}{ Forward } & Reverse \\
\hline Gli-1 & 5'-TCCTTTGGGGTCCAGCCTTG-3' & 5'-ATGCCTGTGGAGTTGGGGCT-3' \\
Gli-2 & 5'-ACGCTAAGTGGCAGTCCTGT-3' & 5'-TGGGGCAGCGAGACTAAATA-3' \\
Gli-3 & 5'-GGGGACAAAGATGAAAGCAA-3' & 5'-GCTTTGAACGGTTTCTGCTC-3' \\
Shh & 5'-CGCACGGGGACAGCTCGGA AGT-3' & 5'-CTGCGCGGCCCTCGTAGTGC-3' \\
SMO & 5'-TTACCTTCAGCTGCCACTTCTACG-3' & 5'-GCCTTGGCAATCATCTTGCTCTTC-3' \\
GAPDH & 5'-GAAGGTGAAGGTCGGAGT-3' & 5'-GAAGATGGTGATGGGATTTC-3'
\end{tabular}

Gli, Glioma-associated oncogene; Shh, Sonic hedgehog; SMO, Smoothened; GAPDH, glyceraldehyde-3-phosphate dehydrogenase.
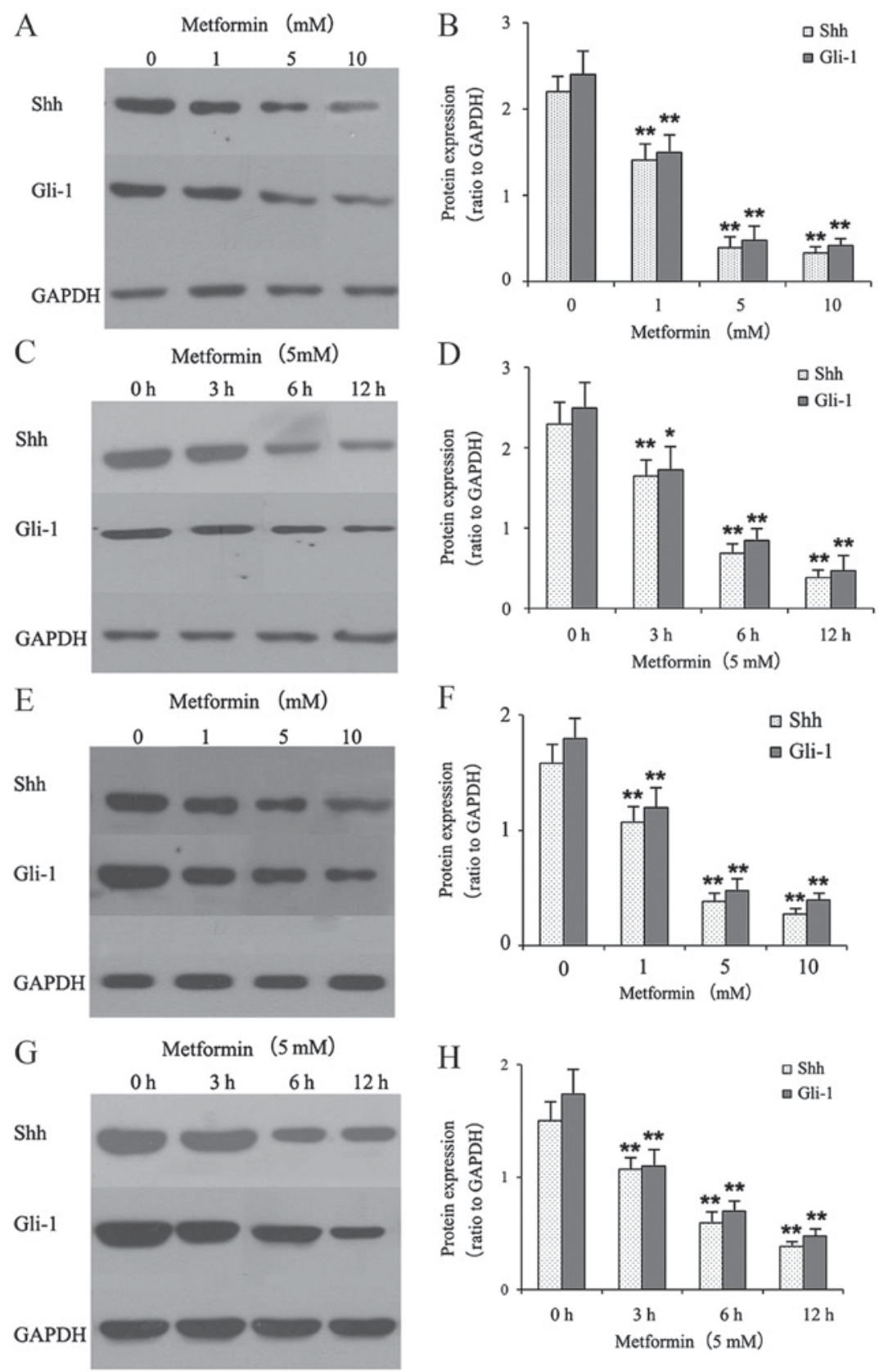

Figure 1. Metformin suppresses protein levels of Shh and Gli-1 in gastric cancer cells. (A and B) HGC-27 cells were treated with metformin at concentrations of $0,1,5$ or $10 \mathrm{mM}$ for $12 \mathrm{~h}$, or with (C and D) $5 \mathrm{mM}$ of metformin for $0,3,6$ and $12 \mathrm{~h}$. (E-H) MNK-45 cells were treated in the same manner. The protein levels of Shh and Gli-1 were measured using western blot analysis; GAPDH was used as a control. Histograms represent the relative protein levels to those of GAPDH. All data are presented as the mean \pm standard deviation $(\mathrm{n}=4)$. " $\mathrm{P}<0.05$, vs. control group; ${ }^{* *} \mathrm{P}<0.01$, vs. control group. Shh, Sonic hedgehog; Gli-1, Glioma-associated oncogene homolog-1. 

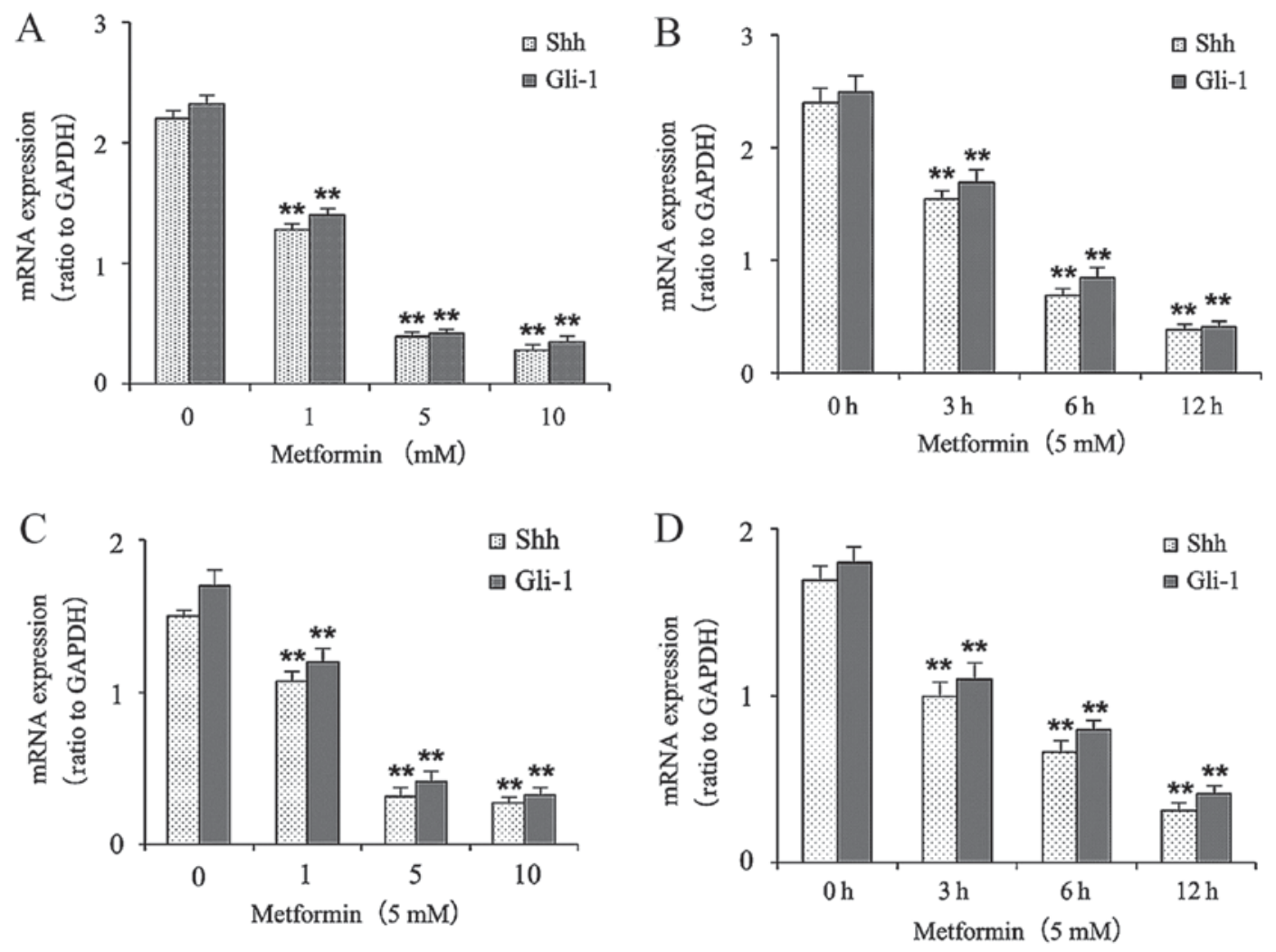

Figure 2. Metformin suppresses mRNA expression levels of Shh and Gli-1 in gastric cancer cells. (A and B) HGC-27 cells and (C and D) MNK-45 cells were treated with metformin at concentrations of $0,1,5$ or $10 \mathrm{mM}$ for $12 \mathrm{~h}$, or with $5 \mathrm{mM}$ of metformin for $0,3,6$ and $12 \mathrm{~h}$. The mRNA expression levels of Shh and Gli-1 were determined using reverse transcription-quantitative polymerase chain reaction analysis. GAPDH was used as a control. Histograms represent the relative mRNA levels to those of GAPDH. All data are presented as the mean \pm standard deviation $(n=4)$. ${ }^{*} \mathrm{P}<0.05$, vs. control; ${ }^{* *} \mathrm{P}<0.01$, vs. control. Shh, Sonic hedgehog; Gli-1, Glioma-associated oncogene homolog-1.

whereas metformin significantly inhibited the upregulation of Gli-1 induced by rhShh. The depletion of AMPK induced by siRNA reversed the suppressive effect of metformin on the rhShh-induced expression of Gli-1in the HGC-27 gastric cancer cells (Fig. 3B and C).

Metformin decreases the gene and protein expression levels of Gli-2, Gli-3 and SMO in gastric cancer cells. The effects of metformin on the gene and protein expression levels of other hedgehog pathway components in the gastric cancer HGC-27 cells, including Gli-2, Gli-3 and SMO, are shown in Fig. 4. As shown in Fig. 4A, treatment with metformin at a concentration of $5 \mathrm{mM}$ for $12 \mathrm{~h}$ exhibited significant decreases in the mRNA expression levels of Gli-2, Gli-3 and SMO, compared with the control group, determined using RT-qPCR analysis $(\mathrm{P}<0.01)$. The results of the western blot analysis (Fig. 4B) demonstrated that $5 \mathrm{mM}$ metformin treatment for $12 \mathrm{~h}$ induced significant decreases in the protein expression levels of Gli-2, Gli-3 and SMO, compared with the control group $(\mathrm{P}<0.05)$.

\section{Discussion}

In the present study, it was demonstrated that metformin significantly inhibited the Shh signaling pathway in gastric cancer cells. It was also shown that AMPK was involved in suppression of the Shh signaling pathway by metformin in gastric cancer cells.
As the leading cause of cancer-associated mortality worldwide, although there have been advances in the treatment of gastric cancer, the prognosis of gastric cancer remains poor $(1,2)$. Thus, there is an urgent requirement to examine novel strategies for the prevention and treatment of gastric cancer. Metformin is generally accepted as an anti-diabetic drug and is also considered a first-line treatment for type 2 diabetes (16). However, in previous years, numerous studies have confirmed that patients treated with metformin have lower incidence and mortality rates of various types of cancer, including lung cancer, breast cancer, pancreatic cancer and gastric cancer (5-8). A study by Kim et al (7) reported that diabetic patients with no administration of insulin had a decreased incidence of gastric cancer when treated with metformin, compared with those who were not treated with metformin. Another previous study showed that the use of metformin as a single agent induced the activation and phosphorylation of mitogen-activated-protein-kinase through the increased heterodimerization of C-RAF/B-RAF in non-small cell lung carcinoma cells (17). In addition, it has been demonstrated that the duration of metformin treatment is associated with the decreased risk of gastric cancer, particularly in patients who used metformin for $>3$ years (7).

However, the mechanism underlying the anticancer effects of metformin remain to be fully elucidated Previous studies have shown that, despite the complexity of the molecular signaling mechanisms of metformin, several critical molecules 

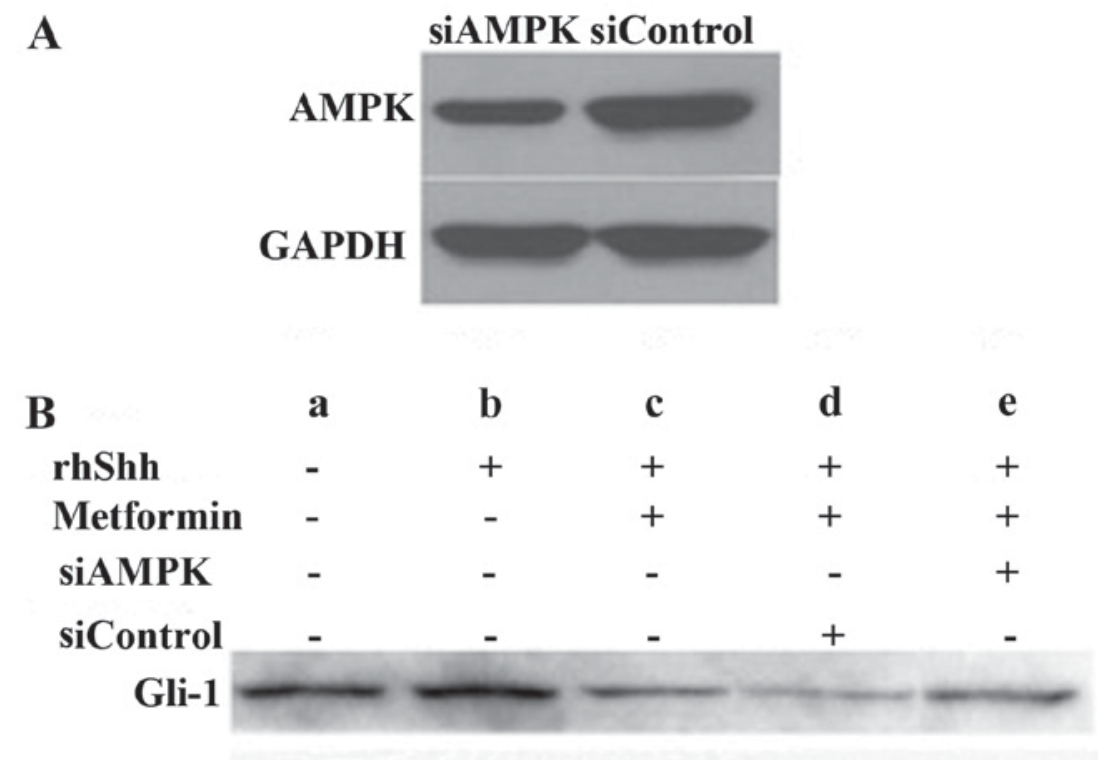

GAPDH

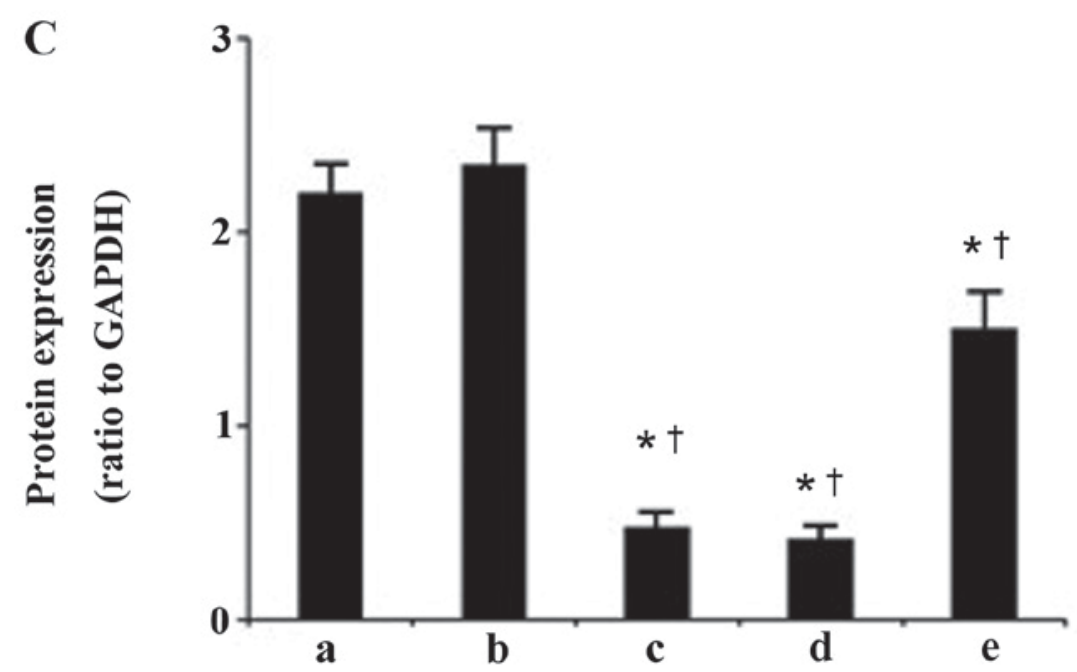

Figure 3. AMPK is involved in the suppressive effect of metformin on the expression of Gli-1. (A) HGC-27 cells were transfected with siAMPK or with siControl. At $48 \mathrm{~h}$ post-transfection, the cells were lysed and examined using western blot analysis; $\beta$-actin was used as aloading control. (B) Cells were treated with (a) PBS alone as a blank control. Untransfected cells were treated with (b) rhShhor the (c) combination of rhShh and metformin. HCG-27 cells transfected with (d) siControlor (e) siAMPK were treated with rhShhor the combination of rhShh and metformin. Protein levels of Gli-1 were examined using western blot analysis. GAPDH was used as a control. (C) Statistical comparisons of protein expression in the five experimental groups ( $\mathrm{n}=4$ ). ${ }^{*} \mathrm{P}<0.05$, vs. (a) group; ${ }^{\dagger} \mathrm{P}<0.05$, vs. (b) group. rhShh, recombinant Sonic hedgehog; Gli-1, Glioma-associated oncogene homolog-1; AMPK, AMP-activated protein kinase; siAMPK, small interfering RNA specific to AMPK.

and pathways have been confirmed to be involved in this regulation, including the activation of $\operatorname{AMPK}(8,18)$, inhibition of mammalian target of rapamycin complex-1 (19), interruption of the crosstalk between insulin and insulin-like growth factor-1 receptor (20) and reductions in the expression of vascular endothelial growth factor and plasminogen activator inhibitor-1 (21).

The Shh signaling pathway is well accepted as being key in numerous cellular processes controlling oncogenesis, angiogenesis and cancer development $(9,10)$. Previous evidence has demonstrated that abnormal activation and/or overexpression of Shh protein is involved in the genesis of gastric cancer (11). Regulation of the Shh signaling pathway may serve as a novel treatment approach for gastric cancer.
A previous study showed that metformin reduced the expression of Shh in pancreatic cancer cells (22). Although the use of metformin has been associated with a reduction of gastric cancer, the effects of metformin on the Shh signaling pathway have not been reported previously. The present study showed that treatment with metformin decreased the expression levels of Shh and SMO, and their downstream molecules, Gli-1, Gli-2 and Gli-3, in gastric cancer cells at the mRNA and protein levels. These results indicated that inhibition of the Shh signaling pathway is one of the underlying mechanisms of the anticancer effects of metformin.

As Shh binds to the transmembrane receptor, Patched, another transmembrane protein, SMO, is depressed (23). Consequently, theGli transcription factors, predominantly 

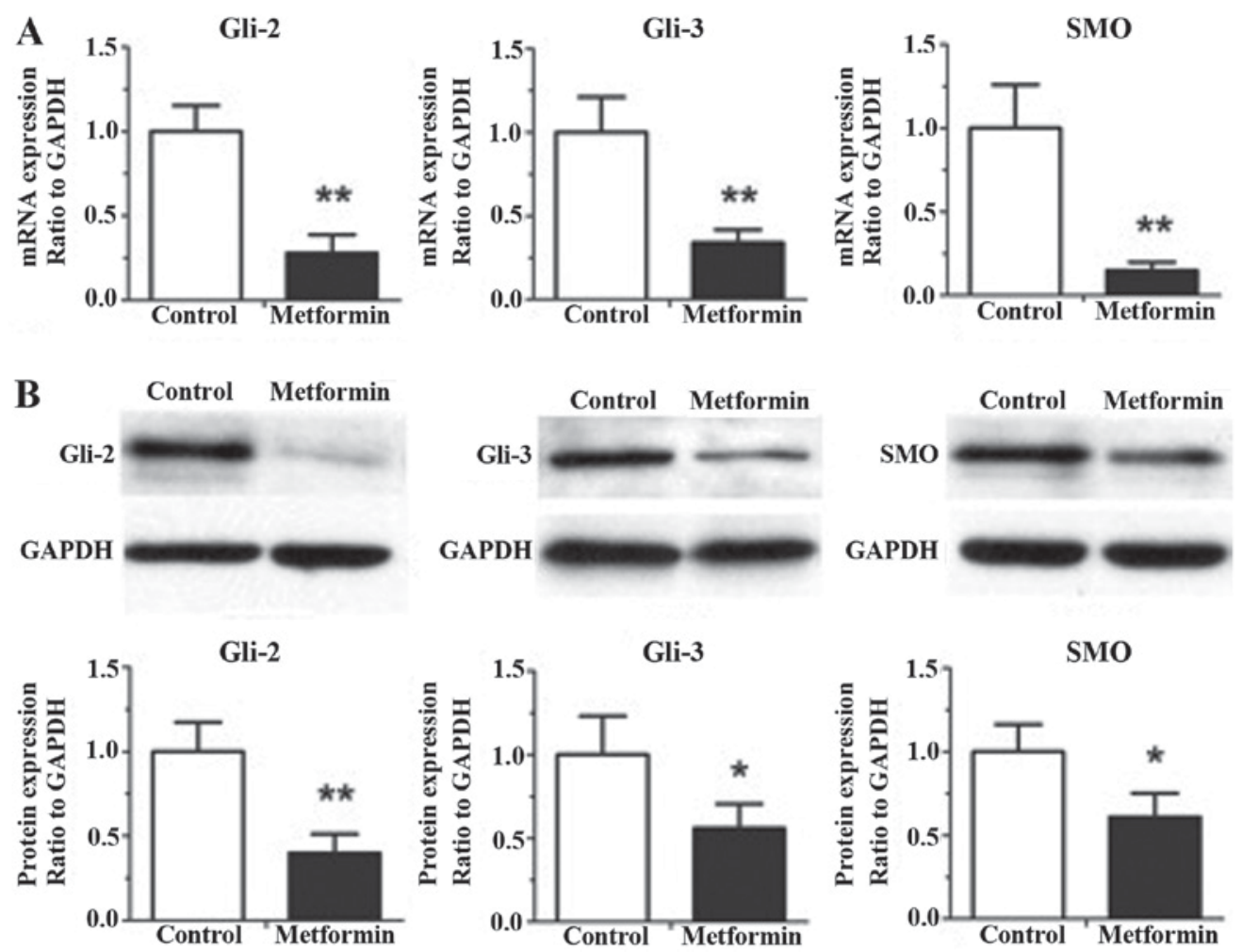

Figure 4. Metformin inhibits the gene and protein expression of other hedgehog pathway components in gastric cancer cells, including Gli-2, Gli-3 and SMO. Gastric cancer HGC-27 cells were treated with metformin at a concentration of $5 \mathrm{mM}$ for $12 \mathrm{~h}$. The (A) mRNA and (B) protein expression levels of Gli-2, Gli-3 and SMO were determined using reverse transcription-quantitative polymerase chain reaction and western blot analyses, respectively. GAPDH was used as a control. Histograms represent the relative mRNA levels to those of GAPDH. All data are presented as the mean \pm standard deviation $(n=4)$. "P<0.05, vs. control group; ${ }^{* *} \mathrm{P}<0.01$, vs. control group. Gli, Glioma-associated oncogene; SMO, Smoothened.

Gli-1, are activated (23). Gli-1 is a potent positive activator of downstream target genes (24). Thus, Gli-1 was considered a marker of abnormal activation of the Shh signaling pathway in the present study.

As the activation of AMPK has been considered as one important mechanism of the anticancer effects of metformin $(8,18)$, the present study investigated whether the suppression of the Shh signaling pathway induced by metformin was AMPK-dependent. Firstly, it was demonstrated that the expression of AMPK was suppressed by siRNA specific to AMPK. The cells were then treated with rhShh and metformin. Compared with untransfected cells or cells transfected with control siRNA, the expression of Gli-1 induced by rhShh was significantly increased in the cells transfected with siRNA specific to AMPK. These results indicated that the presence of AMPK was essential in metformin-induced inhibition of the Shh signaling pathway.

In conclusion, the findings of the present study demonstrated that metformin regulated the expression of Shh in gastric cancer cells, and that inhibition of the Shh signaling pathway by metformin was, at least in part, dependent on AMPK. Further investigations are required to examine the mechanisms underlying the association between the anticancer effects of metformin and the Shh signaling pathway.

\section{Acknowledgements}

The authors would like to acknowledge the support from the National Natural Science Foundation of China (grant no. 81172368) and WuJieping Medical Foundation of China (grant no. 32067001203).

\section{References}

1. Jemal A, Bray F, Center MM, Ferlay J, Ward E and Forman D: Global cancer statistics. CA Cancer J Clin 61: 69-90, 2011.

2. Nagini S: Carcinoma of the stomach: A review of epidemiology, pathogenesis, molecular genetics and chemoprevention. World $\mathrm{J}$ Gastrointest Oncol 4: 156-169, 2012.

3. Lee MS, Hsu CC, Wahlqvist ML, Tsai HN, Chang YH and Huang YC: Type 2 diabetes increases and metformin reduces total, colorectal, liver and pancreatic cancer incidences in Taiwanese: A representative population prospective cohort study of 800,000 individuals. BMC Cancer 11: 20, 2011.

4. Micic D, Cvijovic G, Trajkovic V, Duntas LH and Polovina S: Metformin: Its emerging role in oncology. Hormones (Athens) 10: $5-15,2011$.

5. Evans JM, Donnelly LA, Emslie-Smith AM, Alessi DR and Morris AD: Metformin and reduced risk of cancer in diabetic patients. BMJ 330: 1304-1305, 2005.

6. Zakikhani M, Dowling R, Fantus IG, Sonenberg N and Pollak M: Metformin is an AMP kinase-dependent growth inhibitor for breast cancer cells. Cancer Res 66: 10269-10273, 2006.

7. Kim YI, Kim SY, Cho SJ, Park JH, Choi IJ, Lee YJ, Lee EK, Kook MC, Kim CG, Ryu KW and Kim YW: Long-term metformin use reduces gastric cancer risk in type 2 diabetics without insulin treatment: A nationwide cohort study. Aliment Pharmacol Ther 39: 854-63, 2014.

8. Vallianou NG, Evangelopoulos A and Kazazis C: Metformin and cancer. Rev Diabet Stud 10: 228-235, 2013.

9. Varjosalo M and Taipale J: Hedgehog signaling. J Cell Sci 120: 3-6, 2007

10. Ingham PW: Hedgehog signaling. Cold Spring Harb Perspect Biol 4 pii: a011221, 2012.

11. Pasca di Magliano M and Hebrok M: Hedgehog signalling in cancer formation and maintenance. Nat Rev Cancer 3: 903-911, 2003. 
12. Suzuki H, Minegishi Y, Nomoto Y, Ota T, Masaoka T, van den Brink GR and Hibi T: Down-regulation of a morphogen (sonic hedgehog) gradient in the gastric epithelium of helicobacter pylori-infected mongolian gerbils. J Pathol 206: 186-197, 2005.

13. Berman DM, Karhadkar SS, Maitra A, Montes De Oca R, Gerstenblith MR, Briggs K, Parker AR, Shimada Y, Eshleman JR, et al: Widespread requirement for Hedgehog ligand stimulation in growth of digestive tract tumours. Nature 425 : 846-851, 2003

14. Ma X, Chen K, Huang S, Zhang X, Adegboyega PA, Evers BM, Zhang $\mathrm{H}$ and Xie J: Frequent activation of the hedgehog pathway in advanced gastric adenocarcinomas. Carcinogenesis 26 $1698-1705,2005$

15. Livak KJ and Schmittgen TD: Analysis of relative gene expression data using real-time quantitative PCR and the 2(-Delta Delta C(T)) Method. Methods 25: 402-408, 2001.

16. Alexander GC, Sehgal NL, Moloney RM and Stafford RS: National trends in treatment of type 2 diabetes mellitus, 1994-2007. Arch Intern Med 168: 2088-2094, 2008.

17. Della Corte CM, Ciaramella V, Di Mauro C, Castellone MD, Papaccio F, Fasano M, Sasso FC, Martinelli E, Troiani T, De Vita F, et al: Metformin increases antitumor activity of MEK inhibitors through GLI1 downregulation in LKB1 positive human NSCLC cancer cells. Oncotarget 7: 4265-4278, 2016

18. Zheng L, Yang W, Wu F, Wang C, Yu L, Tang L, Qiu B, Li Y, Guo L, Wu M, et al: Prognostic significance of AMPK activation and therapeutic effects of metformin in hepatocellular carcinoma. Clin Cancer Res 19: 5372-5380, 2013.
19. Sinnett-Smith J, Kisfalvi K, Kui R and Rozengurt E: Metformin inhibition of mTORC1 activation, DNA synthesis and proliferation in pancreatic cancer cells: Dependence on glucose concentration and role of AMPK. Biochem Biophys Res Commun 430: 352-357, 2013.

20. Rozengurt E, Sinnett-Smith J and Kisfalvi K: Crosstalk between insulin/insulin-like growth factor-1 receptors and $\mathrm{G}$ protein-coupled receptor signaling systems: A novel target for the antidiabetic drug metformin in pancreatic cancer. Clin Cancer Res 16: 2505-2511, 2010.

21. Ersoy C, Kiyici S, Budak F, Oral B, Guclu M, Duran C, Selimoglu H, Erturk E, Tuncel E and Imamoglu S: The effect of metformin treatment on VEGF and PAI-1 levels in obese type 2 diabetic patients. Diabetes Res Clin Pract 81: 56-60, 2008.

22. Nakamura M, Ogo A, Yamura M, Yamaguchi $\mathrm{Y}$ and Nakashima H: Metformin suppresses sonic hedgehog expression in pancreatic cancer cells. Anticancer Res 34: 1765-1769, 2014.

23. Ingham PW and McMahon AP: Hedgehog signaling in animal development: Paradigms and principles. Genes Dev 15: 3059-3087, 2001.

24. Lee J, Platt KA, Censullo P and Ruiz i Altaba A: Gli1 is a target of Sonic hedgehog that induces ventral neural tube development. Development 124: 2537-2552, 1997. 